\title{
Haciendo memoria: Vicisitudes de un proyecto de conservación de biodiversidad en el Pacífico colombiano, 1992-19981
}

\author{
Conservation Memories: Vicissitudes of a Biodiversity Conservation Project in \\ the Pacific Lowlands of Colombia, 1992-1998
}

DOI: $10.5935 / 2237-2717.20160008$

\author{
Claudia Leal \\ Universidad de los Andes \\ claleal@uniandes.edu.co \\ Bogotá \\ Colombia
}

Invitada por el editor

\section{RESUMEN}

Este artículo, basado recuerdos y conversaciones con los protagonistas, discute un punto de quiebre en la larga historia de conservación de la naturaleza. A mediados de la década de 1990, tras la Cumbre de la Tierra en Río, las iniciativas de conservación buscaron involucrar a las comunidades locales e ir más allá de las áreas protegidas. Este artículo examina el Proyecto Biopacífico, financiado por el Fondo Mundial para el Medio Ambiente de las Naciones Unidas, que se desarrolló en la costa pacífica de Colombia, territorio habitado por gente negra. Examina las complejidades de hacer realidad el principio de participación de las organizaciones y comunidades locales, en el contexto del giro multicultural latinoamericano. También analiza las distintas interpretaciones que ha tenido esta experiencia, y cuestiona las formas como nuestras maneras de ver el mundo como académicos moldean las historias que narramos.

\section{PaLABRAS CLAVES}

Conservación, biodiversidad, Pacífico colombiano, movimiento negro.

\section{ABSTRACT}

Based primarily on personal memories and interviews, this article discusses a turning point in the long history of nature conservation as it played out in one of the biodiversity hotspots of South America. In the mid-1990s, after the Earth Summit in Rio, conservation initiatives moved beyond protected areas and called for the participation of local communities. The article examines Proyecto Biopacífico, the first biodiversity conservation project financed by the Global Environment Facility, which unfolded in the very humid jungles of the Pacific coast of Colombia - an area inhabited primarily by black people. It unpacks the complexities of involving local communities and organizations in the context of the multicultural turn in Latin American politics. It also analyzes different interpretations of this particular experience of incorporating communities into a conservation project, questioning the ways in which our worldviews, as scholars, often lead us uncritically to find what we are looking for.

\section{KEYWORDS}

Biodiversity conservation, Pacific coast of Colombia, black social movement.

\footnotetext{
1 Traducción de "Conservation Memories: Vicissitudes of a Biodiversity Conservation Project in the Rainforests of Colombia, 1992-1998", Environmental History Vol.20, n.3, 2015 (pp.368-395), realizada por Matías Godoy y revisada por la autora. Quisiera agradecer muy especialmente a los amigos y conocidos que recordaron a Biopacífico conmigo. También estoy agradecida con Emily Wakild, Marisol de la Cadena y Claudia Steiner por sus comentarios sobre una versión previa de este artículo; con los dos evaluadores de Environmental History por sus excelentes sugerencias; con Catalina Muñoz por compartir lecturas e ideas sobre memoria e historia oral, y con Lisa Brady, Graeme Wynn y Jó Klanovicz por creer que este ensayo tiene lugar en una revista académica. Agradezco también a Arturo Escobar, a quien envié una versión preliminar del artículo y me respondió que lo importante es que ambos pateamos el balón en la misma dirección, y a Peter Wilshusen, con quien después de publicado el artículo sostuve la más amable y enriquecedora conversación. Gracias a ella hay algunos cambios sutiles entre las versiones en inglés y en español.
} 


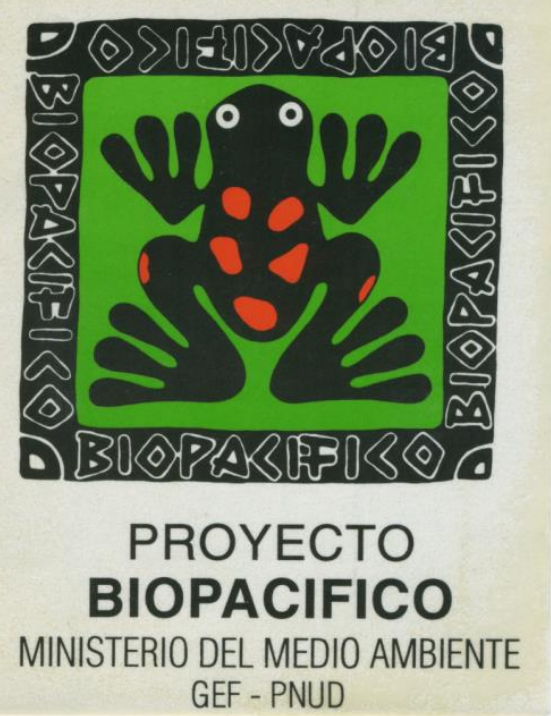

Figura 1 - Logo del Proyecto Biopacífico. Cortesía de Juan Manuel Navarrete.

Concluida la reunión, fuimos a tomar el vuelo de regreso a Bogotá. Era una típica noche caliente en Cali. Fernando Gast (en adelante Fernando G.) compró unas bolitas de caucho en una maquinita y nos entregó una a Enrique Sánchez y otra a mí; caminamos en silencio hacia la puerta de embarque haciéndolas rebotar. Llevábamos más de dos años trabajado juntos en el Proyecto Biopacífico, el primer proyecto financiado por el Fondo Mundial para el Medio Ambiente de las Naciones Unidas (GEF por su nombre en inglés: Global Environment Facility). Su objetivo era producir en tres años la base para una estrategia de desarrollo sostenible y de conservación de la biodiversidad en las selvas del Pacífico colombiano, una de las regiones más biodiversas del mundo. Era febrero de 1996; acabábamos de culminar una innovadora experiencia de ocho meses que buscaba redefinir el proyecto con la plena participación de las organizaciones que representaban a las comunidades negras e indígenas de la región. Este proceso surgió a raíz de una evaluación que determinó que Biopacífico no había cumplido con uno de sus principios básicos: trabajar con las comunidades locales. El día anterior, en la reunión final, cuando aún no habíamos logrado llegar a un acuerdo, el administrador del proyecto había anunciado que ya poco quedaba del fondo de nueve millones de dólares con el que operábamos. Dada la necesidad urgente de concluir el proceso, dividimos el dinero restante entre los grupos que hacían parte de la negociación. Todos compartíamos dos premisas: valorábamos la naturaleza y veíamos en los habitantes locales la clave del futuro de la región. Sin embargo, terminamos actuando como políticos de la peor ralea: repartiéndonos los restos del botín. Ni Fernando G., ni Enrique ni yo necesitábamos palabras para expresar nuestra profunda desilusión.

Han pasado veinte años y todạvía siento la frustración que nos produjo no haber estado a la altura de la tarea que aceptamos con tanto ahínco. Buscando entender qué pasó con Biopacífico, leí parte del último libro de Arturo Escobar, reconocido estudioso del Pacífico que trabaja en Estados Unidos, y un artículo de Peter Wilshusen, profesor de estudios ambientales en la Universidad de Bucknell, que fue uno de los dos autores del informe de evaluación final del proyecto. ${ }^{2}$ Sus miradas son similares y pueden resumirse en

\footnotetext{
${ }^{2}$ Arturo Escobar, Territorios de diferencia (Popayán: Envión Editores, 2010), 152-171; Peter R. Wilshusen, "Territory, Nature, and Culture. Negotiating the Boundaries of Biodivērsity Conservation in Colombia's Pacific Costal Region," en Contested Nature: Promoting International Biodiversity Conservation with Social Justice in the Twenty-first Century, ed., Steven R.
} 
la aserción de Wilshusen según la cual la participación tardía de las organizaciones sociales transformó "lo que muchos consideraban un proyecto fallido en un éxito limitado." ${ }^{3}$ Estas lecturas solo aumentaron mi confusión, pues yo recordaba que en vez de rescatar el proyecto, el proceso de negociación lo había dejado en un letargo del que no se recuperó.

Decidí hablar con algunos de los colegas con los que trabajé y con otras personas involucradas con Biopacífico, incluyendo a líderes de las comunidades indígenas y negras, y al primer Ministro del Medio Ambiente. Lo que presento aquí es una narración basada en mis recuerdos, y enriquecida por tales conversaciones, acerca de las dificultades de implementar el principio de participación local. Aunque las experiencias personales moldean nuestro trabajo como académicos, pocas veces involucramos nuestras propias vidas -sobre todo si somos historiadores- en lo que contamos. Mi participación personal le añade un grado de intimidad a este ensayo, y hace de "entrevista" una etiqueta un poco árida para designar parte del método que usé. También dificulta la tarea de caracterizarlo: aunque comparte elementos con las memorias, es más bien una especie de etnografía histórica que intenta explicar un momento clave de la historia global de la conservación tal como se desarrolló en el Pacífico colombiano.

Es usual que a los recuentos de primera mano se les atribuya veracidad histórica. Sin embargo, las reminiscencias son caprichosas. Los psicólogos nos advierten que "recordar se parece más a inventar una historia que a leerla impresa en un libro... Recordar es una actividad que requiere resolver un problema que consiste en dar una versión coherente de un suceso pasado, y la memoria es la solución a ese problema". ${ }^{4}$ Conservamos ciertos recuerdos y los entretejemos formando historias que satisfacen nuestras necesidades personales y colectivas. ${ }^{5}$ La manera en que cuento la historia de Biopacífico también interpreta ante el lector una parte de mi vida en la que trabajé en una región marginada de Colombia. La forma en que Escobar y Wilshusen se refieren a este proyecto contribuye a su imagen de académicos comprometidos con la tarea de construir, o al menos de imaginar, vidas mejores para quienes han estado históricamente en desventaja. Pero nuestro compromiso puede ser engañoso porque a menudo nos lleva a ver la realidad como queremos que sea. La participación no redimió a Biopacífico, como piensan Escobar y Wilshusen, pero tampoco lo condenó, como recordaba yo. Desmenuzar las complejidades de la inclusión de comunidades y organizaciones locales puede ayudarnos a llevar a cabo mejores proyectos y quizás incluso a construir un mejor futuro; también nos permite reflexionar sobre cómo nuestras visiones del mundo afectan los relatos que construimos sin que seamos del todo conscientes de ello.

\footnotetext{
Brechin, Peter R. Wilshusen, Crystal L. Fortwangler y Patrick C. West (Albany: State University of New York Press, 2003); Manuel A. Ríos y Peter Wilshusen, "Informe de evaluación externa final. Proyecto PNUD-GEF COL/92/G31. Proyecto Biopacífico (1992-1998)" (Bogotá: PNUD, GEF, Ministerio del Medio Ambiente, 1999).

${ }^{3}$ Wilshusen, "Territory, Nature and Culture", 4.

${ }^{4}$ John F. Kihlstrom, "Memory, Autobiography, History", Distinguished Lecture presented at the annual meeting of the Rocky Mountain Psychological Association, Tucson, AZ, Abril 2000, consultado el 14 de agosto de 2014 , http://socrates.berkeley.edu/_kihlstrm/rmpa00.htm.

${ }^{5}$ Alessandro Portelli, The Death of Luigi Trastulli and Other Stories: Form and Meaning in Oral History (Albany: State University of New York Press, 1991). Para una mirāda hermosa e inquietante de las memorias personales ver la obra de Brian Friel titulada Faith Healer (Londres: Faber, 1980).
} 


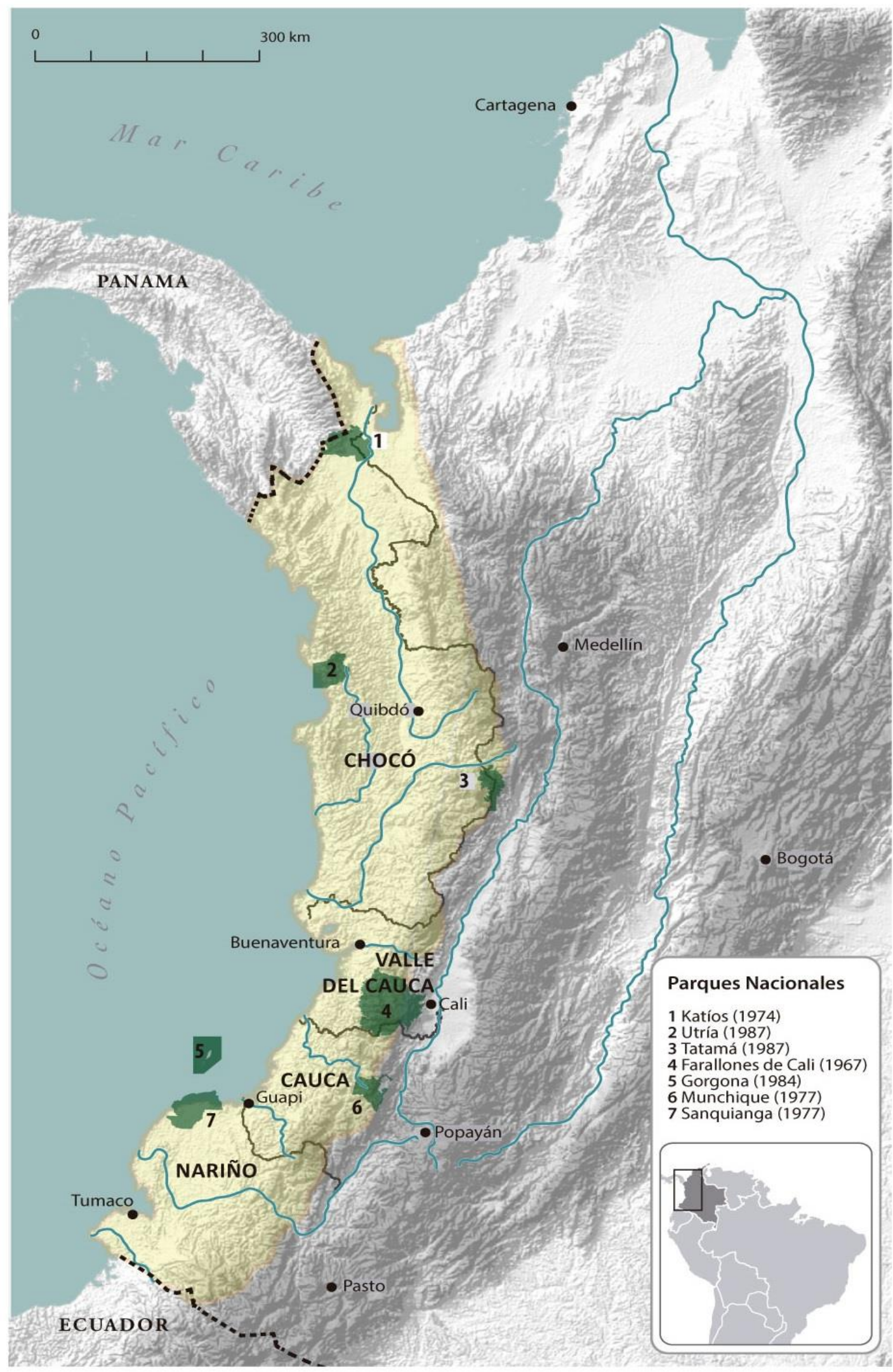

Figura 2 - Area de influencia del Proyecto Biopacífico. Mapa elaborado por Paola Luna, Laboratorio de Cartografía, Universidad de Los Andes, 2015. Basado en el Sistema de Información Geográfica para la Planeación y el Ordenamiento Territorial, Instituto Geográfico Agustín Codazzi, IGAC. 


\section{El comienzo}

Mi primer contacto con Biopacífico fue poco prometedor. Después de graduarme de la universidad trabajé como profesora en una escuelita en la selva a orillas del río Guayabero. Aunque Bogotá quedaba casi en otro planeta y yo no estaba buscando trabajo, una tarde terminé sentada frente a Fernando Casas (en adelante Fernando C.), el coordinador nacional de Biopacífico. Me explicó que necesitaba a un economista que pudiera conversar con altos funcionarios del gobierno; claramente ese no era mi perfil. Volví a la selva y pronto olvidé mi primera entrevista laboral. Unos seis meses después, cuando ya estaba de regreso en Bogotá, Fernando C. me ofreció el cargo. Al parecer, el economista experimentado no existía. Mis credenciales en temas ambientales se limitaban a un par de cursos de biología, mucho interés, y unos cuantos meses en el Amazonas. Que yo haya resultado trabajando en Biopacífico dice mucho sobre cómo el medio ambiente era aún considerado asunto de biólogos: al optar por un camino diferente, el coordinador nacional de Biopacífico tuvo dificultades para encontrar al personal adecuado.

Fui la última en unirme al equipo nacional de Biopacífico, el grupo de profesionales con sede en Bogotá que conformàba las cuatro áreas temáticas con verbos por nombres (idea, al parecer, tomada del Convenio sobre la Diversidad Biológica). Dos biólogos formaban Conocer. Fernando G., con quien compartí aquella noche triste en Cali, tiene un doctorado en biogeografía y estaba a cargo de proporcionar el primer panorama completo de la riqueza biológica de la región. El proyecto debía su existencia a una nueva forma de entender el Pacífico: como megadiverso, más que atrasado. Alwyn Gentry, un reconocido especialista en plantas tropicales, que trabajaba en el Jardín Botánico de Missouri, había contribuido enormemente a ese cambio. ${ }^{6}$ Una etnobotánica entró a reforzar esta área, dado que el proyecto funcionaba bajo el principio de que la biodiversidad existía gracias al manejo y el saber locales. Su tarea era materializar el principio de "diálogo de saberes", que de desprendía de la convicción de la necesidad de tender puentes entre las formas locales y científicas de entender la naturaleza.

En Valorar también éramos dos: Enrique Sánchez, un sociólogo con mucha experiencia, y yo. Tan pronto entré al proyecto Enrique me entregó un informe sobre los sistemas productivos de la población negra de la cuenca del río Atrato. ${ }^{7}$ Así aprendí que nuestra tarea principal era estudiar, junto con las comunidades locales, sus prácticas de manejo del medio ambiente con el fin de mejorarlas, tanto en términos de subsistencia como de sostenibilidad. La idea de fondo era, nuevamente, que la región contaba con extensos bosques gracias a las formas de vida locales y por lo tanto el proyecto tenía que trabajar con y para sus habitantes. Fernando C. también me pidió llevar a cabo un ensayo de valoración económica de la biodiversidad; en esa tarea estaba sola y no sabía por dónde empezar.

Dos personas más trabajaban en Movilizar, la tercera área. Juan Manuel Navarrete fue contratado como especialista en comunicación, al parecer para contarle al mundo lo que estábamos haciendo. Pero él entendió Movilizar como el área sociocultural, no como una herramienta de difusión de lo qué hacían las demás áreas. Su interpretación coincidía con la de Mary Lucía Hurtado, una educadora que había estado involucrada en el movimiento negro que empezó a formarse alrededor de la Constitución de 1991. Mary era la única persona negra y la única de la región trabajando en Bogotá. Ella y Juan Manuel propusieron crear espacios para que los habitantes del Pacífico -tales como profesores, periodistas y

\footnotetext{
${ }^{6}$ Alwyn Gentry, "Species Richness and Floristic Composition of the Choco Region Plant Communities," Caldasia 15 (1986): 71-91; Alwyn Gentry, "Sabemos más de la luna que del Choco'," Eco-Lógica 15-16 (1993): 56-59.

7 July Leesberg y Emperatriz Valencia, "Los sistemāas de producción en el medio Atrato". (Quibdó: Proyecto Diar-

Codechocó, 1987).
} 
operadores de radios comunitarias- definieran lo que para ellos significaba conservación de biodiversidad y lo incorporaran en su trabajo cotidiano. Su idea era escuchar a las personas a las que el proyecto debía beneficiar y permitirles influenciarlo de acuerdo a sus propias necesidades y formas de ver el mundo. ${ }^{8}$ En la última área, Formular-Asignar, Luz Marina Rincón promovía la inclusión de la conservación de la biodiversidad en los planes y programas para la región de la más variada gama de instituciones estatales. En equipo nacional lo completaba Robin Hissong, una especie de secretaria ejecutiva del proyecto.

Ingresé a Biopacífico a finales de 1993 cuando la etapa de planeación estaba terminando, casi un año después del primer intento de dar inicio al proyecto. En febrero de 1993 Fernando C. fue nombrado coordinador nacional. En mayo el equipo ya estaba constituido y trabajaba en su primera tarea: escribir el Plan Operativo que indicaba lo que el proyecto habría de lograr en sus tres años de existencia. Ese periodo había sido concebido como una primera fase, que sería seguida por otra financiada parcialmente por el gobierno colombiano. Al concluir la redacción del Plan Operativo, el equipo de Biopacífico realizó talleres en las tres ciudades principales de la región para informar a las organizaciones e instituciones locales sobre el proyecto y así encontrar futuros socios. En mi primera visita a la región, asistí al último de estos talleres, en Tumaco.

Esos viajes eran muy costosos: el equipo nacional estaba en constante movimiento y Bogotá queda lejos del Pacífico. Por eso al proyecto le pusieron el desafortunado apodo de "Biopaseo". La región no tiene un centro geográfico que sirviera como sede del proyecto, pero Cali habría sido una buena opción: queda a tres horas por carretera de Buenaventura, y está mucho más cerca que Bogotá de Tumaco y Quibdó. Pero el Programa de las Naciones Unidas para el Desarrollo (PNUD), que administraba los fondos del proyecto, y las otras instituciones que lo supervisaban -el Ministerio del Medio Ambiente y el Departamento de Planeación Nacional- funcionan en la capital. Entonces seguí viviendo en Bogotá, pero viajando continuamente a la costa: En esa primera visita a Tumaco (puerto que contaba con menos de cien mil habitantes) escuché a Fernando C. explicar en qué consistía el proyecto a un público adormercido y sentado en un círculo grande. De repente, alguien gritó "iTerapia!" y nos ordenó a todos levantarnos, movernos a la derecha, después a la izquierda y finalmente correr hacia el lado opuesto del círculo y sentarnos. Mientras me ubicaba en mi nuevo puesto vi a una anciana que se había caído y se levantaba lentamente. Todos se reían. Al vernos despiertos y a gusto, el hombre que nos puso a movernos miró a Fernando C. y le dijo: "puede continuar". Era mi primer encuentro con la gente con la que habría de trabajar, y no sabía qué pensar. Ellos tampoco sabían qué pensar del proyecto. Pero los talleres generarron grandes expectativas: nueve millones de dólares eran mucho dinero, especialmente en una región marcada por la pobreza.

${ }^{8}$ Conversación con Juan Manuel Navarrete (Bogotá, 1 de julio de 2013). 


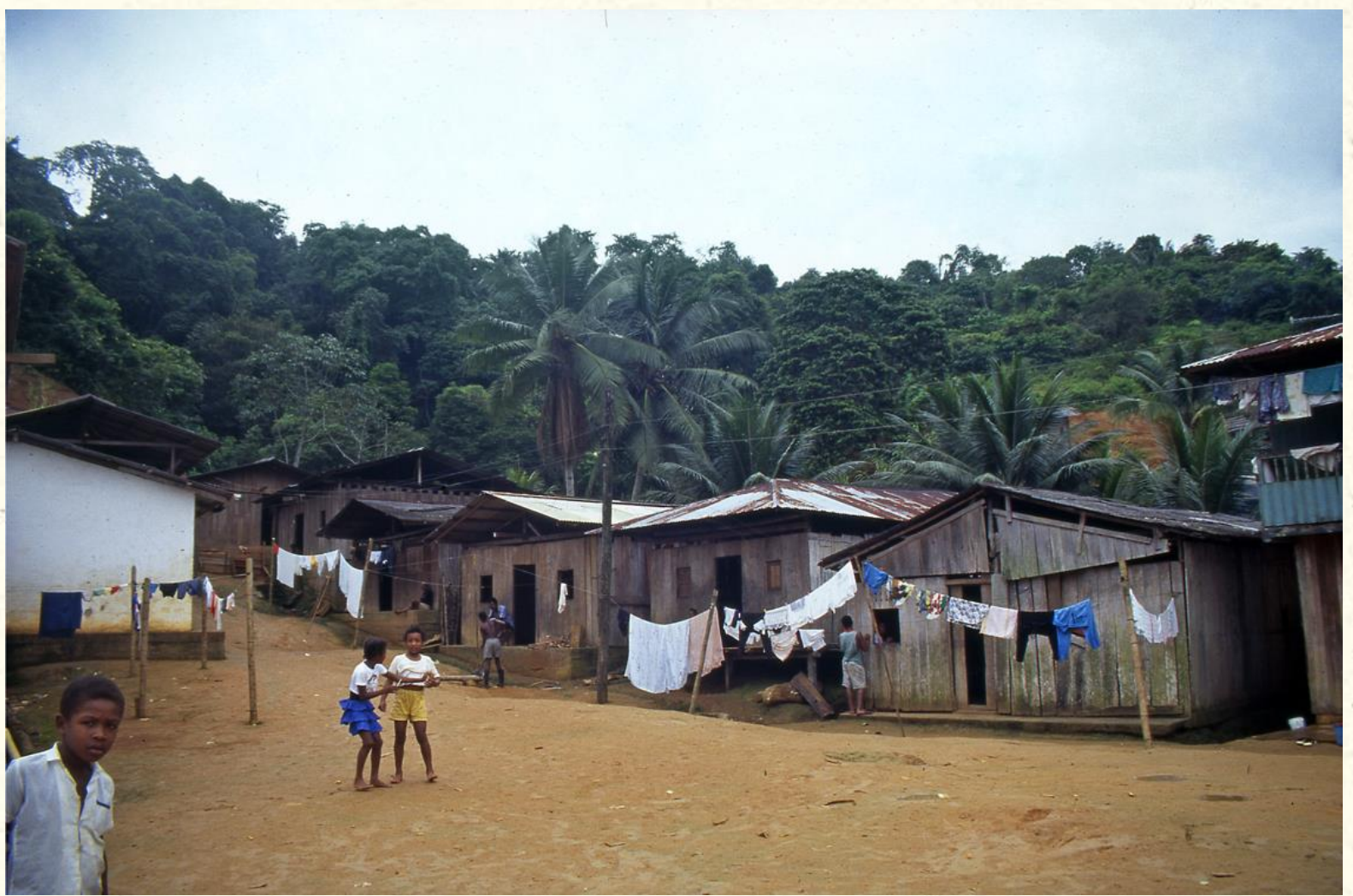

Figura 3 - San Pedro del Vino, Nariño, 1996. Foto de la autora.

Los indicadores sociales del Pacífico han sido siempre peores que los nacionales. En 1993, con 110 muertes por cada mil nacimientos, la tasa de mortalidad infantil casi cuadruplicaba la del país. Ese año la región tenía 2.250.000 habitantes, la gran mayoría negros; cerca del tres por ciento eran indígenas de cuatro etnias diferentes. La mitad de la población vivía en áreas rurales, es decir, en las selvas, que cubrían cerca del ochenta por ciento del territorio. Lluvias inusualmente altas (en algunas partes mayores a 10000 mm por año) y un gradiente altitudinal en el costado occidental de los Andes ayudan a explicar el asombroso número de especies y de endemismo. ${ }^{9}$ Nuestra tarea era producir información, así como generar procesos sociales e institucionales, que permitieran plantear una estrategia para mantener la diversidad natural, y además asegurara mejores condiciones de vida para los habitantes.

\section{Tiempos prometedores, pero inciertos}

Biopacífico es producto del fortalecimiento de las instituciones ambientales a nivel global y nacional. Fue financiado por el GEF, fondo creado por el Banco Mundial y los programas de medio ambiente y desarrollo de Naciones Unidas, a finales de 1991, mientras se planeaba la Cumbre de la Tierra (Río de Janeiro, junio de 1992). El GEF, que tenía como misión fomentar el desarrollo sostenible, privilegió durante sus primeros años la conservación de la biodiversidad sobre sus otras áreas de acción. El término biodiversidad, que hace referencia a la diversidad genética, de especies y de ecosistemas, había surgido recientemente como parte de un esfuerzo mayor por promover la conservación. ${ }^{10}$ Después

\footnotetext{
${ }_{9}^{9}$ Proyecto Biopacífico, Informe Final General, Tomo I: Territorio Biocultural (Bogotá: Proyecto Biopacífico, 1998).

${ }^{10}$ Edward O. Wilson y Frances M. Peter, eds., Biodiversity (Washington, DC: National Academy Press, 1988); David Tacaks, The Idea of Biodiversity: Philosophies of Paradise (Baltimore: The John Hopkins University Press, 1996).
} 
de la Cumbre, el GEF quedó encargado de financiar el Convenio sobre la Diversidad Biológica, uno de los resultados más importantes de aquella reunión.

El GEF representaba una gran oportunidad para Colombia, que ocupa el cuarto puesto en biodiversidad en todo el mundo: tiene el mayor número de especies de pájaros y orquídeas; el segundo de plantas, anfibios y mariposas; el tercero de reptiles; y el cuarto de mamíferos. ${ }^{11}$ Cuando se creó, Claudia Martínez, una colombiana que coordinaba los programas ambientales de Naciones Unidas para América Latina y el Caribe, llamó al director del Instituto Nacional de Recursos Naturales (INDERENA) y le recomendó que presentaran un proyecto de conservación de biodiversidad para el Pacífico. Ella había escuchado que este lugar, al que conocía y le tenía cariño, era el más biodiverso del mundo. Biopacífico fue aprobado en 1992 como el primer proyecto de conservación de biodiversidad del GEF. ${ }^{2}$ A los seis millones de dólares aportados por este fondo, el gobierno suizo le sumó tres más.

La Cumbre de Río promovió una agenda ambiental a nivel global y generó cambios importantes en Colombia. En agosto de 1991, el Departamento Nacional de Planeación aprobó un gran cambio en la política y las instituciones ambientales del país, que en diciembre de 1993 se convirtió en la Ley 99. Eduardo Uribe, miembro de las delegaciones colombianas que fueron a la Cumbre de Río y a las reuniones preparatorias, y primer viceministro del Medio Ambiente, escribió que la participación del gobierno colombiano en la Cumbre "influenció enormemente el contenido de la Ley 99 de 1993 en la medida en que fue una experiencia educativa para los funcionarios del gobierno y congresistas que estaban involucrados en los debates que condujeron a la aprobación de la Ley". ${ }^{13}$ Esta ley, que creó el ministerio del Medio Ambiente, reconoce tal influencia al estipular que "el proceso de desarrollo económico y social del país se orientará según los principios universales y del desarrollo sostenible contenidos en la Declaración de Río de Janeiro de junio de 1992 sobre Medio Ambiente y Desarrollo."

Biopacífico debe su existencia a estos cambios pero también sufrió a causa de la incertidumbre que produjeron. La formulación del proyecto y sus primeros dos años de actividades se llevaron a cabo mientras el ministerio del Medio Ambiente fue concebido y creado, lo cual generó demoras y cierta inestabilidad. Pero los mayores retos provenían de la novedosa forma de concebir la conservación que el proyecto representaba. El gobierno colombiano creó un sistema nacional de áreas protegidas que comenzó tímidamente en los años sesenta y creció en los setenta y ochenta. Cuatro de los parques nacionales designados estaban ubicados enteramente dentro de la región Pacífica, mientras que otros tres, ubicados en la cordillera, tenían solo una parte en esta región-(Figura 2). En vez de crear nuevas áreas protegidas, Biopacífico tenía la tarea de contribuir a una estrategia de desarrollo sostenible en una región biológicamente rica y muchísimo más grande que cualquier parque. Por lo tanto, necesitaba trabajar con la población y las instituciones locales. Este enfoque integral tenía mucho sentido, pero era extremadamente ambicioso; además, no había una guía de cómo proceder.

Esta nueva forma de concebir la conservación tomó forma al tiempo que las comunidades negras adquirían nuevos derechos y que se formaba un movimiento social negro. La constitución de 1991 modificó las premisas básicas que gobernaban a la sociedad colombiana para volverla más democrática. Redefinió a la nación como multiétnica

\footnotetext{
${ }^{11}$ Sistema de Información sobre Biodiversidad de Colombia, consultado el 28 de marzo de 2014,

http://www.sibcolombia.net/web/sib/cifras.

${ }^{12}$ Conversación con Claudia Martínez por Skype (1 de julio de 2013).

${ }^{13}$ Eduardo Uribe Botero, "The Evolution of Colombian Environmental Institutions: 1971-2004," Documento CEDE 2005: 04

(Bogotá: Uniandes-CEDE, 2005), 38.
} 
y así reconoció a las numerosas minorías indígenas marginadas a lo largo de la historia, pues sólo ellas, que conforman el cuatro por ciento de la población nacional, eran concebidas entonces como grupos étnicos, esto es, como poseedoras de una cultura ancestral particular, enraizada en un lugar específico, que las diferencia del resto de la nación. No obstante, la constitución incluyó un artículo de última hora que le abría la puerta al reconocimiento oficial de la población rural negra del Pacífico como grupo étnico. El Artículo Transitorio 55 establecía un plazo de dos años para redactar una ley que regulara el proceso de otorgar títulos colectivos sobre la tierra a las comunidades rurales de la Cuenca del Pacífico (que vivían en baldíos de la nación). La idea de títulos colectivos emulaba la situación de las comunidades indígenas, que tenían esos derechos desde la Colonia. La constitución alteró radicalmente el lugar simbólico de las comunidades negras rurales en el país al considerarlas parte del espíritu multiétnico nacional, en contraposición de su imagen como pobres y atrasadas. Es más, su carácter étnico implicaba un reconocimiento de su papel como custodios de la diversidad ambiental del Pacífico. ${ }^{14}$

La movilización generada antes y durante la redacción de la constitución estimuló la formación de un movimiento social negro. En 1990 se reunieron grupos y líderes de todo tipo para definir las exigencias de la gente negra para la Asamblea Constituyente. A medida que se formaban nuevas organizaciones, se estableció un diálogo productivo entre personas de diversas partes del Pacífico. El movimiento emergente intentaba, por primera vez, representar a la gente negra de toda la costa y escogió el reconocimiento de derechos territoriales como su principal bandera. Antes de los años noventa, la movilización política de la gente negra había sido débil y centrada en las poblaciones urbanas. El único grupo con aspiraciones nacionales había sido Cimarrón, una pequeña red de grupos ubicados en varias ciudades del país, dominada por estudiantes universitarios e inspirada por los movimientos de Estados Unidos y Suráfrica en contra de la discriminación racial. Con la nueva constitución, el énfasis pasó de las ciudades a las selvas, y la lucha por la inclusión se empezó a dar desde una perspectiva étnica basada en el derecho a ser diferente. ${ }^{15}$

El Chocó abrió el camino en esta dirección. A mediados de los ochenta los campesinos del río Atrato lucharon contra la expansión de la industria maderera. Aunque habían vivido ahí desde los tiempos de la esclavitud, no tenían títulos sobre sus tierras. Conformaron la Asociación Campesina Integral del Atrato (ACIA) y encontraron argumentos en la convención 169 de la Organización Internacional del Trabajo sobre comunidades indígenas para fortalecer su causa. Siguieron a sus vecinos indígenas quienes, en 1981, formaron la Organización Regional Embera y Waunan (Orewa), y quienes habían logrado que les titularan extensos resguardos. A medida que se desarrollaban los preparativos para la Asamblea Constituyente, este modelo de organización comunitaria se extendió a otros ríos del Chocó y también hacia el sur. ${ }^{16}$ Allá, sin embargo, el proceso resultó un poco diferente, pues sus líderes fueron jóvenes profesionales urbanos.

La experiencia de Mary Lucía Hurtado (coordinadora del área sociocultural de Biopacífico) ayuda a comprender ese proceso. Nacida y criada en el río Timbiquí, Mary fue a la universidad en Cali donde, después de estudiar para ser profesora, hizo una maestría en educación que culminó con una tesis ên la que analizó cómo los pensum de los colegios del Pacífico ignoraban la cultura regional. En Cali se unió a Cimarrón y a las discusiones sobre las experiencias de sus miembros con el racismo. Poco después ingresó al equipo del

\footnotetext{
${ }^{14}$ Kiran Asher, Black and Green: Afro-Colombians, Development, and Nature in the Pacific Lowlands (Durham: Duke University Press, 2009).

${ }^{15}$ Carlos Efrén Agudelo, Retos del multiculturalismo en Colombia (Medellín: La Carreta, IRD, ICANH, Universidad Nacional de Colombia, 2005); Asher, Black and Green

${ }^{16}$ Eduardo Restrepo, Etnización de la negridad: la invención de las comunidades negras como grupo étnico en Colombia (Popayán: Universidad del Cauca, 2013).
} 
proyecto de desarrollo más importante del Pacífico hasta el momento (Plaidecop) donde, bajo la orientación del sociólogo Gustavo de Roux, empezó a pensar sobre desarrollo y participación. Fueron esos días de creciente concientización política, en los que Mary colaboró con el "movimiento de 1991" que había surgido sorpresivamente. Combinó sus roles como funcionaria y activista al organizar talleres a lo largo de los ríos de la región para discutir temas como los derechos políticos y la cultura regional. Este tipo de esfuerzos crearon lazos entre esa dirección en formación ubicada en el puerto de Buenaventura y los habitantes de los ríos. Era una época -recuerda Mary- en que "no había una estructura orgánica fuerte, era más un proceso de confluencia de muchos actores". ${ }^{17}$

El intento de crear un movimiento para toda la región fracasó, puesto que las organizaciones campesinas del Chocó tomaron su propio camino y mantuvieron distancia con la dirección urbana del sur. A finales de 1993, cuando la ruptura se hizo oficial, los entusiastas jóvenes profesionales de Buenaventura crearon el Proceso de Comunidades Negras (PCN), el cual, según sus líderes, incluía "una red de más de ciento veinte organizaciones locales." 18 De la mano de la Iglesia y otros aliados estratégicos ayudaron a los pobladores de cada uno de los ríos del sur a organizarse. ${ }^{19}$

A medida que iban surgiendo organizaciones, se formó la comisión que redactó la ley definida por el Artículo Transitorio 55, cuyo objetivo era regular la titulación comunal de tierras a las poblaciones rurales del Pacífico. Entre agosto de 1992 y mayo de 1993, la comisión trabajó en lo que sería la Ley 70, adoptada en agosto de 1993. Representantes comunitarios, junto con funcionarios del gobierno, redactaron la histórica ley al tiempo que el recién formado equipo de Biopacífico definía las directrices del proyecto. Algunas de las reuniones de la comisión ocurrieron en la sede de Biopacífico, de manera que sus miembros y el equipo nacional del proyecto tuvieron la oportunidad de tener más de una conversación. Una vez el Congreso aprobó la ley, líderes comunitarios y sus aliados organizaron talleres informativos a lo largo de la región, mientras Biopacífico empezaba su fase de implementación. Entretanto, la autoridad ambiental nacional aseguraba fondos para el proceso de titulación al incluirlo en un préstamo que el gobierno negociaba con el Banco Mundial. Manuel Rodríguez, el primer ministro del Medio Ambiente, y artífice de este resultado, está convencido de que sin tales fondos la ley se habría convertido en letra muerta. ${ }^{20}$ Con la ley en una mano y una preocupación oficial por el medio ambiente en la otra, los líderes del incipiente movimiento social negro, al igual que muchos otros quienes, como yo, habíamos llegado a trabajar a la costa en ese momento, creíamos que este era el comienzo de una nueva era para la región. Este gran optimismo marcó la percepción que todos teníamos sobre Biopacífico.

\section{Socios y proyectos}

Desde los inicios en Nueva York, Bogotá y Cali, se buscó desarrollar un proyecto de conservación de la biodiversidad con y para las comunidades locales. El concepto mismo de biodiversidad, con sus connotaçiones científicas, sugería que se trataba de una

\footnotetext{
${ }^{17}$ Conversación con Mary Lucía Hurtado (Bogotá, 22 de enero de 2014).

${ }^{18}$ Libia Grueso, Carlos Rosero y Arturo Escobar, "El proceso de organización de comunidades negras en la región sureña de la Costa Pacífica de Colombia", en Política cultural, cultura política. Una nueva mirada sobre los movimientos sociales latinoamericanos, eds., Sonia E. Álvarez, Evelyn Dagnino y Arturo Escobar (Bogotá: Taurus-ICANH, 2001), 235-260; ver también Asher, Black and Green, 54.

${ }^{19}$ Eduardo Restrepo, "Imaginando comunidad negra: etnografía de la etnización de las poblaciones negras en el Pacífico sur colombiano," en Acción colectiva, Estado y etnicidad en el Pacífico colombiano, ed., Mauricio Pardo (Bogotá: ICANH Colciencias, 2001), 41-70; Restrepo, Etnización dê la negridad.

${ }_{20}$ Conversación con Manuel Rodríguez (Bogotá, 30 de septiembre de 2013).
} 
propuesta estrictamente biológica, lo que llevó a algunos, entre ellos al botánico Alwyn Gentry, a pensar que el objetivo del proyecto era realizar un gran inventario biológico. ${ }^{21} \mathrm{Sin}$ embargo, esta interpretación resultaba insostenible en el contexto colombiano de principios de los noventa. La biodiversidad fue pronto entendida como riqueza natural y su existencia explicada por la presencia histórica de las comunidades negras e indígenas; así, no se trató de una noción estrictamente biológica, sino también profundamente social y política. Además, el GEF requería que sus proyectos fueran participativos; pero nadie sabía cómo hacer realidad esta directriz. Por lo tanto, más que un requisito, la participación era una meta. Así, Biopacífico fue un gran laboratorio para la conceptualización y la materialización del polémico asunto de la participación.

Para hacer de Biopacífico mucho más que una iniciativa científica, Claudia Martínez, quien redactó la propuesta presentada al GEF, buscó a profesionales que conocieran el lugar y a sus habitantes, así como a expertos en sus aves y plantas. Encontró a un buen socio en Gustavo de Roux, el reconocido sociólogo caleño que tenía una amplia experiencia de trabajo con comunidades negras tanto en la costa como en el norte del Cauca. De Roux defendía la idea de que el proyecto debía fortalecer la capacidad de las comunidades locales para controlar sus territorios frente a intereses tales como los del capital minero y maderero. ${ }^{22}$ En esta fase inicial de planeación no participó nadie de la región, en parte porque los profesionales del Pacífico eran poco reconocidos a nivel nacional. En esos años solo dos universidades operaban en toda la costa, y su énfasis estaba puesto en la educación técnica. ${ }^{23}$ Para conocer la opinión de quienes ella consideraba los principales beneficiarios del proyecto, Martínez organizó un taller en Guapi. En esta reunión, personas del pueblo y sus alrededores buscaron expresar -en parte a través de canciones y poemaslo que para ellos podía significar la biodiversidad. El uso de talleres para diseñar proyectos no era una práctica usual, y para Martínez fue difícil que Naciones Unidas le reembolsara los gastos en los que incurrió.

A pesar de estos esfuerzos, al analizar la propuestá, Alberto Galán concluyó que el proceso se había llevado a cabo a un ritmo de Nueva York que no permitía una participación comunitaria adecuada. Galán, quien estaba terminando un doctorado en políticas ambientales en Estados Unidos, realizó su análisis por solicitud de The Nature Conservancy. El trabajo con líderes negros realizado dentro de un grupo de derechos humanos le proporcionó un conocimiento incipiente sobre la problemática de las comunidades negras de la costa que resultó fundamental para su lectura del proyecto. ${ }^{24}$

Una vez el GEF aprobó la propuesta, Manuel Rodríguez fue, primero como jefe del INDERENA y después como primer ministro del Medio Ambiente, el responsable de hacer que el proyecto arrancara. Su primer candidato para coordinador nacional no duró y el segundo, Gustavo de Roux, no aceptó, pues consideraba que la oficina principal debía estar en Cali. El tercero, Fernando C., gozaba de un manejo excepcional de la diplomacia ambiental pero apenas conocía la costa. Conformó un equipo que, con un par de excepciones, replicaba su falta de experiencia en la región. Este equipo redactó el Plan Operativo sin plena conciencia de la contradicción que implicaba su objetivo principal: "Aportar para la región colombiana" del Chocó biogeográfico elementos que permitan consolidar una nueva estrategia de desarrollo basada en la aplicación del conocimiento

\footnotetext{
${ }^{21}$ Para una crítica del concepto de biodiversidad, ver Escobar, Territorios de diferencia, 158-163.

${ }^{22}$ Conversación telefónica con Gustavo de Roux (Bogotá-Cali, 12 de octubre de 2013).

${ }^{23}$ Universidad Tecnológica del Chocó en Quibdó (1975) y el campus de la Universidad del Valle en Buenaventrua (1986).

${ }^{24}$ Conversación con Alberto,Galán (Bogotá, 15 de octubre de 2013).
} 
científico y la identificación de opciones de manejo de la biodiversidad que garanticen su protección y su uso sostenible, en forma concertada con las comunidades locales." 25

Tal "concertación", al parecer, no incluía la concepción del proyecto. Esta manera de proceder seguía una larga tradición a la que Libia Grueso (miembro de Biopacífico y también líder del PCN) se refirió como "colonial". ${ }^{26}$ Con el fin de involucrar a las comunidades y a otros grupos de interés, Biopacífico envió un borrador del Plan Operativo a 150 organizaciones comunitarias y no gubernamentales, universidades y agencias del estado, cuyos comentarios fueron incorporados en la versión final. ${ }^{27}$ A pesar de su ausencia en la etapa de planeación, la meta de incluir a las comunidades locales no era un simple saludo a la bandera. La participación tomó dos formas iniciales: la inclusión de personas de la región y de los movimientos sociales en el equipo del proyecto, y el trabajo conjunto con comunidades y organizaciones locales.

Con la notable excepción de Mary, las personas de la región fueron contratadas para trabajar en las oficinas regionales creadas a mediados de 1993, cuando el Plan Operativo recibía sus últimos retoques. El proceso de contratación de coordinadores regionales fue meticuloso; eran personas respetadas y, en los dos sitios en donde el movimiento social era más fuerte, representaban al movimiento o tenían su aval. Jairo Miguel Guerra fue nombrado en Chocó con el apoyo tanto de la OREWA como de la ACIA, las organizaciones indígena y negra más importantes de esta área. Aunque es de la Costa Caribe, Jairo estaba (y sigue estando) casado con una médica chocoana y había trabajado en el Chocó durante muchos años como sacerdote y con la OREWA. Alberto Achito, uno de los líderes más antiguos de esta organización, lo considera uno de sus mentores. ${ }^{28}$ El conocido poeta Alfredo Vanín, oriundo del río Saija, ocupó el cargo en Nariño. Fernando C. decidió apropiadamente que alguien de la organización emergente de Buenaventura debía ocupar la oficina regional en ese puerto. Recibió tres nominaciones entre las que eligió a Libia Grueso, una aguerrida trabajadora social. La cuarta oficina regional, ubicada en Guapi, abrió mucho después.

Como lo demuestran sus nombramientos tardíos, los coordinadores regionales no tuvieron participación alguna en la concepción del Plan Operativo. Esto los situaba en una posición subordinada que pronto generó tensión. Jairo y Libia se dieron cuenta muy pronto de que su papel era principalmente el de operadores logísticos para planes y actividades concebidos en Bogotá, y ninguno de ellos estaba dispuesto a trabajar en esas condiciones. Jairo recuerda una reunión que se llevó a cabo antes de que yo entrara al proyecto, en la cual los coordinadores regionales, con el apoyo de algunos miembros del equipo nacional, le dijeron a Fernando C. que esa situación era insostenible. De ahí en adelante las cosas empezaron a cambiar lentamente. ${ }^{29}$ Los coordinadores regionales gozaban de ciertas ventajas: conocían su área de operaciones, eran necesarios para la ejecución del proyecto y además ayudaban a legitimarlo. Pero para alterar su posición debían formar equipos fuertes y llegar a un entendimiento propio sobre lo que significaba la conservación de la biodiversidad y cómo podía traducirse en acciones concretas. Aprovechando su experiencia en la lucha contra la posible construcción de un puerto en Bahía Málaga, Libia, por ejemplo, interpretó la misión del proyecto como la protección de los territorios negros frente a amenazas externas. ${ }^{30}$

\footnotetext{
${ }^{25}$ El énfasis es mío.

${ }^{26}$ Conversación con Libia Grueso (Bogotá, 9 de agosto de 2013).

${ }^{27}$ Wilshusen, "Territory, Nature, and Culture".

${ }^{28}$ Conversación con Alberto Achito (Bogotá, 29 de enero de 2014).

${ }^{29}$ Conversación con Jairo Miguel Guerra (Bogotá, 25 de junio de 2013).

${ }^{30}$ Conversación con Libia Grueso.
} 
Los coordinadores regionales eran vitales para la segunda forma de hacer realidad la participación: el trabajo con las comunidades locales y sus organizaciones. Ellos tenían la responsabilidad de forjar relaciones con líderes y gente de la región para trabajar alrededor de intereses comunes. Aunque el área Movilizar fue probablemente la que más logró en este respecto, voy a centrarme en mi propia experiencia. Mi colega, Enrique, había definido los sistemas locales de producción como nuestro objetivo principal. Debido a su experiencia en la región, fue invitado a participar en discusiones, que antecedieron el nombramiento del coordinador nacional, sobre las metas del proyecto. Allí explicó que los sistemas de producción eran la forma en que los habitantes locales entendían y manejaban el medio ambiente y garantizaban su bienestar, y propuso estudiarlos para determinar cómo podían fortalecer tanto la conservación como el bienestar de las comunidades. Aunque esta propuesta encontró cierta oposición, fue respaldada por una misión temprana de Naciones Unidas y posteriormente por el equipo nacional, y así llegó a ocupar un lugar destacado en el Plan Operativo. ${ }^{31}$ Nuestra idea era llevar a cabo, y en la medida de lo posible también contratar, los estudios con organizaciones comunitarias.

En el Chocó, gracias al entusiasmo de Jairo y a la fortaleza de las organizaciones y ONGs, este trabajo avanzó bastante bien. La ACIA decidió que debíamos realizar un estudio en Tanguí, para lo cual requeríamos de un agrónomo o un antropólogo que trabajara hombro a hombro con la comunidad y redactara el informe final. Sabíamos que encontrar a un chocoano para llevar a cabo esta tarea sería mucho más difícil que conseguir a alguien de Bogotá o Medellín, pero lo primero tenía más sentido pues contribuiría a construir experticia local. Odilio Cuesta, un joven economista chocoano (que había estudiado en Medellín y no tenía experiencia en el área rural) asumió esta responsabilidad.

La Fundación Natura, quizás la ONG ambiental más sólida del país, llevó a cabo otra de estas evaluaciones en el Chocó, sobre la base de varios años de trabajo en el río Valle con la organización local, Asproval. Natura redactó un excelente informe y sintetizamos los resultados en una cartilla. ${ }^{32}$ Además, junto con Jairo, organizamos en Quibdó una reunión regional sobre la economía de las comunidades rurales del Pacífico, cuyas memorias publicamos y distribuimos. ${ }^{33}$ Este encuentro pionero -que reunió a agrónomos que trabajaban en la región y a miembros de organizaciones locales, entre otros- contribuyó a validar la idea de que el conocimiento local era necesario para resolver los problemas locales. También ayudó a construir una red de personas, organizaciones e instituciones dedicadas a temas similares pero en lugares distantes.

En otras partes de la región tuvimos menos suerte con nuestras investigaciones sobre sistemas productivos locales. En Nariño comenzamos a trabajar con Coagropacífico, una cooperativa campesina, pero esta iniciativa terminó cuando uno de sus líderes se gastó en asuntos personales (por corrupción o para lidiar con una emergencia) el dinero que Biopacífico había adelantado. Nuestro segundo intento, más corto que el primero, ocurrió en el río Saija. Biopacífico había contratado a una persona que vivía en Guapi para ayudar con asuntos logísticos en la costa caucana. Durante nuestra visita al río, destinada a iniciar conversaciones con la gente local, dicha persona sacó a relucir un lamentable alcoholismo y así caímos en cuenta de que para proseguir necesitábamos una verdadera oficina regional. En Valle no tuvimos iniciativas en los primeros años.

\footnotetext{
${ }^{31}$ Conversación con Enrique Sánchez (Bogotá, 25 de junio de 2013).

${ }^{32}$ Carlos Tapia, Rocío Polanco y Claudia Leal, Los sistemas productivos de la comunidad negra del río Valle, Bahía, Solano, Chocó (Bogotá: Proyecto Biopacífico, 1997).

${ }^{33}$ Claudia Leal, ed., Economía de las comunidades rurales en el Pacífico colombiano (Bogotá: Proyecto Biopacífico, 1995).
} 
Trabajar con las comunidades requería de atención constante que solo podían ofrecer las oficinas regionales. El éxito de Jairo provino en parte de la creación de una oficina con seis empleados, de los cuales tres eran profesionales. Aún así tuvieron muchas dificultades, tales como navegar las turbulentas aguas de la política comunitaria y resolver innumerables problemas logísticos. Contratar a ONGs, universidades o investigadores individuales era mucho más fácil y podíamos hacerlo desde Bogotá, como en efecto ocurrió a menudo sin consultarlo con las comunidades donde se llevarían a cabo los proyectos. Buena parte de este tipo de contratación la hizo Conocer, el área a cargo de proveer el primer mapa de la biodiversidad de la región, para lo cual debía reunir todo el conocimiento científico disponible pero disperso, además de producir información nueva. Fernando G. trabajó con biólogos para organizar una base de datos y para usar bioindicadores (tales como aves rapaces) que permitieran hacer evaluaciones rápidas del estado de la biodiversidad. Esta no era una tarea fácil, pero los biólogos sabían cómo hacer este tipo de trabajo. Por este motivo, en el primer año de implementación del proyecto, esta área temática fue la que más presupuesto ejecutó. ${ }^{34}$

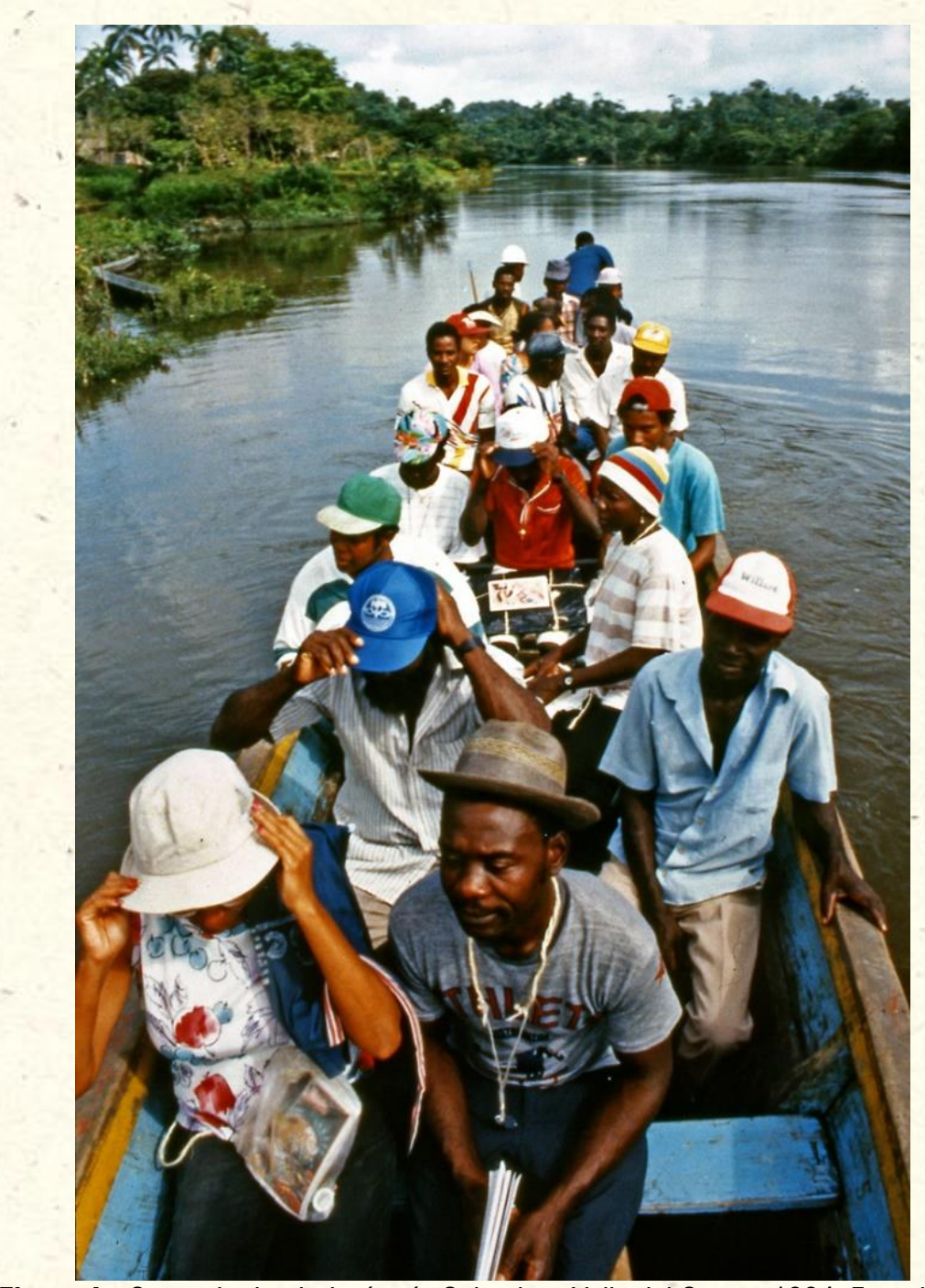

Figura 4 - Curso de dendrología, río Cajambre, Valle del Cauca, 1994. Foto de la autora.

\footnotetext{
${ }^{34}$ Wilshusen, "Territory, Nature, and Culture."
} 
Fernando G. no tenía experiencia en hacer estudios con comunidades locales, sin embargo tuvo una idea original. Buscó a Gilberto Mahecha, un experimentado ingeniero forestal de origen campesino que había ideado un método para identificar árboles en campo mirando su forma, corteza, hojas y otras características no reproductivas (dado que las flores, usadas para la clasificación científica, suelen estar ausentes). Aunque este método no era el más valorado por el establecimiento científico, Fernando G. entendió su importancia y potencial. ${ }^{35}$ El trabajo de Mahecha consistió en realizar cursos (de dendrología) de una semana con gente local para volver realidad nuestro principio de intercambio entre conocimiento local y científico. Los asistentes (en grupos de veinte más o menos) empezaron a entender la lógica de clasificación científica y además se sorprendieron de ver cuánto sabía Mahecha sobre las plantas locales, a pesar de ser del interior del país. Un manual de campo que explica este método y una cartilla sobre de las plantas de Tanguí (que también incluía una sección sobre la historia de la comunidad) fueron parte de los resultados de esta iniciativa ${ }^{36}$ Libia, que era muy crítica del énfasis científico de Biopacífico, habla de estos cursos como uno de los éxitos del proyecto: "Fue impresionantemente exitoso, la gente desde ahí se quedó haciendo inventarios de especies en los territorios étnicos como patrimonio de las comunidades". ${ }^{37}$

Biopacífico era mucho más que los ejemplos que he presentado aquí. Con un objetivo muy ambicioso y sin mayor dirección por parte del coordinador nacional, era más una serie de empeños medianamente conectados que un todo coherente. También sufría de tensiones internas, en gran parte producto de las diferentes nociones sobre lo que debía ser la participación local. Juan Manuel y Mary, desde el área Movilizar, sentían que la necesidad de trabajar con la gente, a su ritmo, no había sido comprendida del todo. Según Kiran Asher, en el momento estudiante de doctorado y hoy profesora de Clark University, Mary pensaba que "Biopacífico... simplemente decidió que los movimientos sociales y los procesos políticos en la región no eran asunto suyo". 38 Pero la persona más contrariada por la forma de trabajar de Biopacífico era Libia, quien como ya dije no solo era la coordinadora regional en el Valle sino también líder del PCN. Ella, al igual que su organización, creía que Biopacífico no estaba trabajando realmente con las organizaciones negras, las cuales según la Ley 70 debían ser parte del proceso de toma de decisiones. Pensó en renunciar en protesta por el modus operandi de Biopacífico, mientras algunos de sus colegas hacían lobby en el ministerio del Medio Ambiente para corregir la situación.

\section{¿Cambio radical?}

En 1995 una misión de evaluación determinó que había que reformular el proyecto con la plena participación de las organizaciones que representaban a las comunidades negras e indígenas. Julio Tresierra, un profesor peruano que vivía en Canadá y había sido contratado por el PNUD, encabezó la misión, de la que también hacían parte dos colombianos y un suizo. La misión giró en torno a una serie de talleres públicos realizados en varias partes de la región para escuchar la opinión que la gente tenía sobre Biopacífico. Kiran Asher asistió al taller en Buenaventura y escribió:

\footnotetext{
${ }^{35}$ Conversación con Fernando Gast (Bogotá, 29 de junio de 2013).

${ }^{36}$ Proyecto Biopacífico, Algunos árboles y otras plantas de Tanguí (Bogotá: Proyecto Biopacífico, 1993); Gilberto Emilio Mahecha Vega, Fundamentos y metodología para la identificación de plantas (Bogotá: Proyecto Biopacífico, 1997).

${ }^{37}$ Conversación con Libia Grueso.

${ }^{38}$ Asher, Black and Green, 7.6.
} 
La opinión entre los miembros de la comunidad negra era generalizada y consistente: la participación y el respeto por los derechos étnicos rara vez trascendía el nivel retórico en las prácticas del Proyecto Biopacífico y otras entidades del estado [...] La participación local en temas de desarrollo y biodiversidad estaba por lo general restringida a informar a las comunidades sobre los planes actuales y propuestos para la región o a incorporar gente local como empleados rasos, asistentes o informantes en proyectos de investigación. ${ }^{39}$

Los miembros de la misión de evaluación no cuestionaron sus limitaciones para interpretar la política local y no se tomaron la molestia de hablar con muchos de los miembros del equiipo de Biopacífico, incluyéndonos a Enrique y a mí, que conformábamos el área socioeconómica. Juan Manuel Navarrete, que había luchado por incluir a la gente de la región más plenamente en el proyecto, ni siquiera recuerda la misión. ${ }^{40}$

Pero sí recuerda sus consecuencias. Creamos un "Equipo Ampliado", conformado por el equipo nacional y los coordinadores regionales de Biopacífico, y representantes de organizaciones negras e indígenas, para redactar un nuevo Plan Operativo. Nos reunimos tres veces en ocho meses y no logramos definir una nueva serie de prioridades. Este viraje ocurrió cuando Biopacífico estaba por empezar su última etapa. Aunque era claro que el proyecto iba a durar más de los tres años que habían sido estipulados originalmente, también era cierto que para mediados de 1995 habían transcurrido dos años y medio, y muchos de los recursos financieros se habían gastado. Dado que estábamos definiendo nuevas directrices, se detuvieron todas las contrataciones nuevas (a excepción de proyectos muy pequeños) y Biopacífico entró en un período de letargo.

En nuestra primera reunión en Perico Negro me correspondió presentar las propuestas del equipo nacional, pero en lugar de animar la discusión, terminé acusada de perpetuar el modelo centralizado que debíamos superar. Este tipo de comportamiento anclado en la denuncia, comúnmente usado para tratar a los funcionarios del estado (que no era exactamente nuestro caso), marcó el tono de las conversaciones, lo que nos molestó a muchos. En esta reunión inicial, los representantes del movimiento negro afirmaron que solo podían hacer propuestas en conjunto con las comunidades locales y pidieron tiempo y recursos para hacer talleres. Así que unos meses pasaron antes de nuestra siguiente -y muy tensa- reunión en Piangua Grande, en la cual los representantes de las comunidades negras nos sorprendieron diciendo que no podían revelar àsuntos discutidos en sus talleres, pues eso violaría su autonomía.

La discusión que siguió se enfocó en principios y produjo, como bien lo explican Wilshusen y Escobar, una nueva definición de biodiversidad y una nueva meta general para Biopacífico:

El Proyecto Biopacífico propenderá por la construcción de una estrategia de conocimiento, uso, manejo y conservación de la biodiversidad en el territorio-región del Pacífico colombiano que se inserte en las políticas locales, regionales y nacionales de desarrollo de manera que permita la defensa de la cultura y el patrimonio biológico de la Nación y de los grupos étnicos del Pacífico colombiano y la construcción de modelos alternativos de desarrollo. ${ }^{41}$

\footnotetext{
${ }^{39}$ Asher, Black and Green, 74-75.

${ }^{40}$ Conversación con Juan Manuel Navarrete.

${ }^{41}$ Escobar, Territorios de diferencia; Wilshusen, "Territory, Nature, and Culture."
} 
Cómo traducir tales principios en acciones concretas era todo un misterio. No recuerdo ninguna discusión sobre cómo revitalizar el área socioeconómica. La OREWA, la organización indígena, empezó la negociación con un proyecto ya definido, al cual llamaban Plan de Vida, que consistía en estudiar las amenazas a sus territorios para planear su manejo futuro. Los representantes de las comunidades negras concentraron sus energías en discusiones abstractas. Su trabajo, además, no era remunerado. Como me dijo Víctor Guevara, representante de Buenaventura en el Equipo Ampliado, "todo eso fue sacado a pulso y con hambre." Víctor pasó largas noches estudiando los documentos de Biopacífico para entender el proyecto, esforzándose en definir posiciones y propuestas. ${ }^{42}$ El Equipo Ampliado sin duda operó bajo circunstancias muy desiguales. Finalmente, el nuevo Plan Operativo no incluyó propuestas novedosas; más bien cambió el peso relativo de elementos ya existentes y otorgó un lugar más central a las comunidades locales.

Redefinir el proyecto con la plena participación de las comunidades locales tuvo otras dificultades centradas en el asunto fundamental de quién tenía derecho a representarlas. En el Chocó, esta cuestión no produjo mayor controversia. La OREWA agrupaba a todas las comunidades indígenas, y como la ACIA era la organización negra más fuerte, acabó representando a las demás. En el sur estaba el PCN, organización que buscaba tener alcance regional al agrupar a todas las demás organizaciones. Pero su legitimidad tenía límites, por lo menos en dos niveles. Primero, sus líderes, estacionados en la ciudad de Buenaventura, eran de origen urbano y en muchos casos profesionales, mientras que los líderes de los ríos eran campesinos que no siempre querían que personas de la ciudad hablaran en su nombre. Sin embargo, la alianza entre ellos era mutuamente beneficiosa: el apoyo local le permitía a la dirección del PCN establecerse como representante legítima de las comunidades ribereñas en sus tratos con el estado, y de esta forma le daban voz política a grupos previamente marginados. Segundo, el modelo funcionaba bien en Valle, pero no en todas partes. El tema era particularmente problemático en Cauca, donde la comunidad se estaba organizando a su propio ritmo sin presencia real del PCN.

Aunque un poco tarde, Biopacífico estableció una oficina regional en Guapi encabezada por Oscar Alzate, un ingeniero forestal bogotano con buena experiencia en la región. Junto con dos profesionales guapireños -su esposa Aura Elena González, trabajadora social, y Jairo Cuero, un agrónomo- formaron un equipo muy eficiente. De la mano del área sociocultural, construyeron algunas de las relaciones más sólidas de Biopacífico con las comunidades locales. En algunos casos trabajaron directamente con grupos locales que habían emergido en años anteriores gracias a esfuerzos de la Iglesia católica y Plaidecop, el proyecto de desarrollo que mencioné anteriormente; en otros ayudaron a mujeres sobre todo rurales a organizarse. Por ese mismo tiempo, el PCN escogió a CocoCauca como su representante local. Esta organización se había formado en 1994 con la idea de replicar el modelo de representar a todos los grupos locales, pero carecía de liderazgo al punto que surgió una organización rival. ${ }^{43} \mathrm{El}$ resultado de esta situación en el Equipo Ampliado de Biopacífico fue un profundo distanciamiento entre el coordinador regional del Cauca, que defendía el trabajo de Biopacífico como plenamente comunitario, y los representantes del PCN, que acusaban a Biopacífico de obstruir el proceso de construcción de una organización social en el Cauca por no trabajar directamente con sus elegidos.

En la última reunión del Equipo Ampliado, a la que me referí al comienzo de este ensayo, repartimos los fondos sobrantes entre los presentes. Las áreas temáticas recibimos una parte cada una para terminar lo que habíamos empezado. Cada área dentro de la

\footnotetext{
${ }^{42}$ Conversación con Víctor Guevara por Skype (Bōgotá-Buenaventura, 21 de marzo de 2014).

${ }^{43}$ Conversación con Aura Elena González (Bogotá, 26 de junio de 2014).
} 
región recibió una suma para nuevos proyectos que debían ser concebidos conjuntamente entre la oficina regional y las organizaciones, que en el caso del Valle eran una y la misma cosa. Pero en el Cauca, una suma le fue asignada a la oficina regional y otra al PCN. Sin embargo, el representante del PCN del Cauca posteriormente se retiró del Equipo Ampliado porque sus propuestas no fueron aprobadas. Incluso Libia reconoce que sus intereses eran de corte personal. ${ }^{44}$

Biopacífico continuó sus operaciones durante un par de años más. El equipo ampliado aparentemente se mantuvo, pero no hubo ninguna reunión adicional que nos incluyera a todos. En Bogotá el entusiasmo inicial se perdió, y varios de los miembros del equipo nacional salieron del proyecto, especialmente Fernando G. y Mary. Pocos quedaron para escribir el informe final que se publicó en 1998. En las regiones, sobre todo en el Valle, la negociación permitió que el equipo regional y las organizaciones lograran una mayor apropiación de Biopacífico y produjeran resultados concretos.

\section{Interpretaciones divergentes}

La participación comunitaria no es solo deseable sino fundamental para que las iniciativas de conservación sean exitosas, pero no es claro qué significa y cómo se implementa. Antes que nada, la participación es altamente política: la forma en que se define y se lleva a cabo determina quién puede moldear proyectos sociales, cómo y hasta qué punto. El carácter político de la participación también se hace evidente en la manera en que ésta se interpreta, como resulta claro en los relatos sobre el proceso de negociación de Biopacífico.

En sus publicaciones, Wilshusen y Escobar ofrecen versiones simplificadas de un proyecto que, según ellos, evitó naufragar al incorporar tardíamente a las organizaciones locales en el proceso de toma de decisiones. Para ellos, la negociación de 1995-96 marcó un "cambio dramático" sin el cual el proyecto habría fracasado: "De haber mantenido una estrategia dominada por la investigación científica, muy probablemente se habría frustrado ante la resistencia local." " La redención provino del cambio "de la participación marginal de las organizaciones políticas (el movimiento social) a un diseño en que estas ocuparon un lugar cardinal." 46

Pero ninguno de los dos autores explica en qué consistía la participación marginal inicial o las complejidades de implementar este principio. Cuando Biopacífico arrancó, el movimiento social negro estaba formándose. Muchas comunidades no tenían organizaciones que les dieran voz o estaban apenas empezando a crearlas, y la relación entre estos grupos locales y los líderes regionales aún tenía que cuajar. El PCN solo se formó en octubre de 1993 y operaba como una red bastante informal. Incluso en 1995, y especialmente en algunas partes tales como el Cauca, había confusión respecto a quién representaba a la gente que vivía sobre muchos de los ríos que atraviesan la selva. Wilshusen reconoce que Biopacífico "reaccionó ante y estaba restringido por... el rápido crecimiento y maduración de un movimiento social amplio de grupos negros e indígenas que buscaban consolidar y redefinir șus derechos ciudadanos". " 47 in embargo, no explica en qué radicaban tales restricciones y menciona sin comentarlo que los miembros de las comunidades que -participaron en el proceso de negociación decían representar a 684 organizaciones comunitarias. ${ }^{48}$ Que el movimiento fuera tan reciente también significaba que

\footnotetext{
${ }^{44}$ Conversación con Libia Grueso.

${ }^{45}$ Wilshusen, "Territory, Nature, and Culture", 75, 86

${ }^{46}$ Escobar, Territorios de diferencia, 214.

${ }^{47}$ Wilshusen, "Territory, Nature, and Culture", 75-76.

${ }^{48}$ Ibid., 79.
} 
sus tenaces y entusiastas líderes tenían una experiencia limitada. Los más aguerridos habían desarrollado tácticas útiles para oponerse a la reticencia del estado a consultar con las comunidades asuntos que les afectaban, pero estaban mucho menos preparados para proponer o implementar soluciones. Wilshusen y Escobar dan la impresión de que la participación era un asunto muchísimo más sencillo de lo que en realidad era.

Estos autores desperdician la oportunidad de usar el caso de Biopafício para analizar o por lo menos para reconocer que las dinámicas mismas de los movimientos sociales son fundamentales para darle forma a la participación. Y al considerar la negociación como un punto de inflexión enfatizan -y exageran- los cambios ocasionados por este proceso. Escobar reconoce que "más que en los enfoques de biodiversidad convencionales preferidos por ONGs del norte, el PBP hizo constar desde el comienzo la correlación entre la diversidad biológica y cultural." ${ }^{49}$ Añade, sin embargo, que aunque el proyecto abogaba por la participación de las comunidades y la inclusión de conocimientos locales, poco de esto hubo en sus primeros dos años. Según él, "el proyecto pasó por una mutación sólida, de un enfoque científico y administrativo de conservación convencional a un proyecto profundamente negociado que labró una conceptualización alternativa de la biodiversidad." ${ }^{0}$ Como ejemplo de este cambio crucial, Escobar retrata de manera imprecisa la trayectoria del área socioeconómica: "Valorar era para apoyar las iniciativas locales sustentables; en la práctica, esto solamente sucedió hacia el final del proyecto, con una estructura más participativa después de 1995. En su período inicial, la dimensión de "utilización" del proyecto se mantenía economicista, dadas las iniciativas de las ONGs productivistas, y guiada por la necesidad de determinar el valor de la biodiversidad y los costes de oportunidad de su conservación." ${ }^{51}$ Como expliqué anteriormente, el énfasis en sistemas productivos locales fue parte del corazón del proyecto incluso antes de la formulación del Plan Operativo, y aunque Fernando C. me confió la tarea de abrir el camino hacia una valoración económica de la biodiversidad, no logramos firmar un contrato para dar inicio a este proceso. De manera similar, Wilshusen afirma que las oficinas regionales fueron creadas tardíamente, en 1995, cuando de hecho se conformaron en $1993 .{ }^{52}$

¿Por qué estas interpretaciones difieren tanto de la mía? El asunto tiene que ver con el lugar desde el cual cada uno de nosotros conoció el proyecto. Al ser externos a Biopacífico, Escobar y Wilshusen tuvieron menos información de la que tuve yo, sobre todo considerando la naturaleza desarticulada del proyecto y la extensa geografía sobre la que se desarrolló. Además, ellos decidieron privilegiar el punto de vista de las organizaciones sociales negras, en especial sus innovaciones conceptuales. Conocí a Arturo en la sede de Biopacífico en Bogotá, durante una discusión sobre una iniciativa pequeña, financiada por Biopacífico, en la que él estaba involucrado. Me pareció una persona generosa, honesta y dedicada, una impresión que el tiempo sólo ha corroborado. Poco después supe que colaboraba con la dirección del PCN, un lazo que se ha mantenido a lo largo de años y que influyó tanto en las ideas que produjo el PCN como en la forma en que Arturo entiende la historia de Biopacífico. Wilshusen intentó reducir la distancia que lo separaba de estas organizaciones sociales enfocándose, al igual que Escobar, en la propuesta que ellas presentaron en la reunión de Piangua Grande. Estos autores subrayan los conceptos nuevos que el PCN ideó y llevó a la mesa de negociación, particularmente la idea de biodiversidad como intrínsecamente relacionada con el territorio y la cultura. Sus textos dan la impresión que tales propuestas surgen en contraposición a la forma cómo había operado el proyecto, y no reconocen que fueron en buena medida producto del proyecto mismo.

\footnotetext{
${ }^{49}$ Escobar, Territorios de diferencia, 210.

50 lbid., 208.

51 Ibid., 211.

52 Wilshusen, "Territory, Nature, and Culture", 78
} 
Desafiando la idea prevaleciente sostenida por el Convenio sobre Diversidad Biológica, los líderes negros propusieron una noción que elimina la distancia entre los humanos y la naturaleza; para ellos, términos relacionados como monte y territorio indican que el medio ambiente es un espacio vivo que permite la reproducción de la vida material de la gente local y la existencia de prácticas culturales. También explicaron que la biodiversidad no solo tiene un aspecto tangible, sino uno intangible relacionado con las diversas maneras de conocerla. Por último, afirmaron que históricamente las comunidades negras e indígenas han desarrollado relaciones armónicas con el medio ambiente que han permitido la permanencia de la diversidad biológica. ${ }^{53}$

Para otros, como yo, que habíamos estado construyendo Biopacífico y que estuvimos presentes en esas largas reuniones, el tema en cuestión era mucho más práctico; requería ir más allá de definir directrices: se trataba de ponerlas en práctica. En general, las ideas propuestas por la dirección del PCN no encontraron mayor resistencia; eran producto del ethos de la época, y expresaban una mirada común. Una excepción fue la idea de territorioregión, que servía para señalar al Pacífico como el dominio de grupos étnicos y fue considerada por algunos como arrogante y excluyente. ${ }^{54}$ La disputa principal, sin embargo, fue la naturaleza misma de la discusión: quedarse en el plano conceptual o proponer caminos concretos que ayudaran a garantizar la conservación de la naturaleza con bienestar social. Como dice Escobar, "el carácter político de los representantes de los procesos organizativos en el Equipo Ampliado desentonaba a veces con el papel más técnico del Equipo Técnico" ${ }^{55}$ Ellos buscaban establecer una nueva forma de relación con el Estado al tiempo que fortalecían sus organizaciones; nosotros queríamos llevar a Biopacífico a feliz término.

Hay otra razón importante que explica la convicción de Wilshusen y Escobar de que la participación comunitaria enderezó el curso de Biopacífico. Tal convicción ilustra una manera de ver el mundo que aboga por el empoderamiento de grupos en desventaja como forma de construir un futuro mejor. El libro de Escobar opera a un nivel más bien abstracto y gira en torno a la necesidad de crear alternativas a la modernidad a partir de la diferencia, como por ejemplo la mirada presuntamente orgánica de la naturaleza que tienen las comunidades negras de la Costa Pacífica colombiana. El artículo de Wilshusen hace parte de un libro, del que él es coeditor, que promueve la conservación con justicia social. Este libro hace parte de una discusión más amplia que buscaba cuestionar las críticas hechas a los esfuerzos de conservación que incluían objetivos de desarrollo y fuerte participación local, y llamaban a reforzar el proteccionismo autoritario. ${ }^{56}$ En las narraciones que ellos construyen, la exitosa participación de las comunidades organizadas en la definición e implementación de este proyecto les permite sustentar una mirada optimista de los problemas que buscan resolver. Aunque comparto sus ideales, creo que es necesario reconocer y enfrentarse a las complejidades y ambigüedades presentes en tales empeños para mejorarlos. Quizás soy un zorro, como proponía Isaiah Berlin, y me regocijo ante la variedad de experiencias que el mundo ofrece sin pretender mucha coherencia; mientras que Escobar, y quizás Wilshusen también, son erizos, que ven el mundo desde un único principio organizador. ${ }^{57}$

Mis propios recuerdos y mi relato también nacen de mi lugar particular en esta historia. Durante años he creído que Biopacífico funcionaba bien, guiado por un equipo dedicado

\footnotetext{
${ }^{53}$ Escobar, Territorios de diferencia, 217; Wilshusen, "Territory, Nature, and Culture", 80-82.

${ }^{54}$ Escobar, Territorios de diferencia, 76-78; Restrepo, Etnización de la negridad, 211-13.

${ }^{55}$ Escobar, Territorios de diferencia, 214.

${ }^{56}$ Peter Wilshusen, Steven R. Brechin, Crystal L. Fortwangler y Patrick C. West, "Reinventing a Square Wheel: Critique of a

Resurgent "Protection Paradigm" in International Biodiversity Conservation," Society \& Natural Resources 15, (2002): 17-40.

${ }^{57}$ Isaiah Berlin, El erizo y la zorra. Tolstoi y su visión de la historia (Barcelona: Ediciones Península, 2009).
} 
que contaba con el apoyo de socios muy valiosos, hasta que la prolongada negociación lo empantanó, y luego no se logró recuperar. En esta versión, el proceso de negociación sirve como chivo expiatorio para explicar la frustración con la que quedamos muchos miembros del equipo: nos habíamos propuesto salvar un ambiente (y a una gente) y fallamos. Pero aquí nuevamente la realidad es más compleja: las deficiencias de Biopacífico tienen causas mucho más complicadas, y no todo fue decepción. Tuvimos logros considerables, como haber producido y compilado una cantidad de información sin precedentes sobre la región, y haber puesto el medio ambiente en el centro de las discusiones regionales. ${ }^{58}$ Biopacífico también contribuyó a la creación de redes de organizaciones comunitarias y de gente interesada e involucrada con el Pacífico, y finalmente jugó un papel importante en crear la idea de una región interconectada. Además, contribuyó a la formación del movimiento social de las comunidades negras. Un logro notorio en este sentido fue la creación de la red Matamba y Guasá en el Cauca, que reunió a setenta y dos organizaciones de mujeres que trabajaban en la conservación y recuperación de plantas comestibles y medicinales. ${ }^{59}$ Esta red tuvo un papel estratégico al romper el estancamiento al que las dos organizaciones regionales rivales habían llevado el proceso de titulación de territorios comunitarios en esta zona. $^{60}$

No obstante, Biopacífico no estuvo a la altura de las expectativas generadas por el emocionante contexto en que se desarrolló, y la causa no fue el proceso de negociación. Para empezar, las expectativas de lo que esperábamos lograr eran poco realistas. Además, el carácter experimental del proyecto, sumado a su gran escala y reducido marco temporal, creó enormes dificultades. El proyecto debía haber tenido una segunda fase que podría haber superado en parte esta limitación, pero nunca se llevó a cabo, y el legado de Biopacífico se diluyó en el Instituto de Investigaciones Ambientales del Pacífico (IIAP), establecido por la Ley 99 de 1993 y creado en 1997 con sede en la ciudad de Quibdó. La pérdida de importancia de los asuntos ambientales en la década siguiente empeoró la situación. Tales factores adversos señalaban un problema más hondo: en Colombia la investigación no necesariamente sirve de base para la formulación de políticas, de manera que nuestros esfuerzos no tuvieron un fin claro. $Y$ el conflicto armado en Colombia, que hasta entonces no había tenido una presencia tan fuerte en la región, acabó por penetrarla con consecuencias atroces. Se empezó a sembrar coca, y los grupos paramilitares enfrentaron a las guerrillas, generando dolor y violencia. Muchas personas involucradas con Biopacífico sufrieron personalmente las consecuencias de esta tragedia: Jairo (que encabezaba nuestra oficina en Quibdó) y Juana Padilla, la líder en cuya casa solía hospedarme en Tanguí, fueron secuestrados. Por fortuna ambos regresaron sanos y salvos. Varios líderes tuvieron que salir de la región, a otros los mataron, y algunas comunidades resultaron empobrecidas o incluso desplazadas.

El proceso de negociación no fue el culpable de los resultados insatisfactorios de Biopacífico. Aunque en cierto nivel fue una experiencia frustrante, permitió que grupos sociales clave se adueñaran del proyecto, fortaleció la relación entre organizaciones indígenas y negras, y sirvió como escuela para líderes regionales que pulieron sus estrategias para futuras experiencias con instituciones del estado, como la Unidad de Parques Nacionales. Pero sobre todo, fue un privilegiado ejercicio de construcción democrática, en el que perdí parte de mi inocencia política.

\footnotetext{
${ }^{58}$ Ríos y Wilshusen, "Informe de evaluación externa final".

${ }^{59}$ Asher, Black and Green, 145-48.

${ }^{60}$ Conversación con Aura Elena González.
} 